\title{
CONSTRUCTION ET ENTRETIEN DES OUVRAGES D'ÉTANGS DE PISCICULTURE
}

\author{
par H. BOTTON \\ Ingénieur Divisionnaire des Services Techniques de la Ville de Paris
}

Au cours de l'aménagement piscicole deș étangs domaniaux du Der, réalisé en collaboration étroite avec MM. Chimits et Rabouilne, Inspecteurs des Eaux et Forêts, et Kneczyra, Ingénieur Agronome Polonais, nous avons été amené à aborder, avec eux, divers problèmes et à résoudre certaines difficultés pratiques.

Nous pensons que les quelques indications ci-dessous, fruit d un travail commun, pourront rendre service aux propriétaires d'étangs, comme ils ont pu déjà tirer profit des articles de M. Hirsch el de M. Martin, parus dans les numéros antérieurs à ce même Bulletin (n*36, 343,144$)$.

De nombreux étangs utilisés pour la pisciculture sont établis en France en terrains imperméables, marneux ct argileux, sur lesquels l'eau est simplement retenue par une digue en terre.

Le choix de l'emplacement, l'établissement des ouvrages, leur entretien sont des questions très importantes que nous proposons d'examiner. Elles sont fonction de quelques règles générales essentielles qu'il importe de respecter scrupuleusement. Les méconnaitre, c'est s'exposer à de gros risques et à des mécomptes certains qui font que la Pisciculture devient une entreprise onéreuse et que le produit récupéré n'est pas en rapport avec la valeur du fonds.

Quelles sont donc ces règles et comment les ouvrages doivent-ils être conçus ?

Tout d'abord, le terrain qui constituera le sous-sol de l'étang devra être de terre bien imperméable et comporter une arrivée d'eau suffisante pour permettre un remplissage de Janvier à Avril.

Il est utile de ne pas remplir avant Janvier, car cela permet de procéder à un assec hivernal. Cette opération culturale excellente augmente notablement, et sans frais, le rendement. A un autre point de vue, le cours de la Carpe subissant toujours une baisse marquée en Novembre et Décembre, époque où les étangs sont normalement pêchés, il est important - si l'on ne dispose pas d'installation de stockage - de pouvoir effectuer des pêches 
tardives, tout en étant certain de remplir au printemps. Par ailleurs, il faut se réserver, à l'occasion, les derniers mois de lannée, pour exécuter, s'il y a lieu, les réfectibns ì la digue et à la bonde.

Il est préférable que les eaux de ruissellement, alimentant l'étang après chaque pluie, aient drainé des terrains de culture et des prairies, afin d'apporter dans l'étang engrais chimiques ou déjections animales. Les sols des pâtures sont très recommandés car les animaux, en venant à l'abreuvoir, fument l'eau abondamment.

Les dimensions des bassins sont variables el commandent, en principe, les élevages à entreprendre :

- Les petits, de $1 / 4$ à 3 ou 4 hectares, conviennent pour l'alevinage ;

- Les moyens, de 3 à io ou i 5 hectares, conviennent pour les poissons de deux étés ;

- Les grands, de ro à 50 hectares et plus, conviennent pour les poissons de trois étés ou de consommation.

Toutefois, on peut poser, comme règle générale, qu'un bon étang ne doit pas avoir plus de $2 \mathrm{~m}$. 50 de profondeur, car le plancton végétal qui so développe, grâce à l'assimilation chlorophylienne, a besoin de lumière et de chaleur et les rayons lumineux traversent mal les couches d'eau épaisses.

C'est sur la constitution de la digue de l'étang qu'il est intéressant d'attirer l'attention du Pisciculteur. Les marnes et les argiles, sur lesquelles doit reposer l'ouvrage, doivent etre de bonne tenue. Mais, quelles que soient leurs qualités, elles sont éminemment sensibles aux surcharges et se déforment lentement quand s'exerce sur elles une pression d'une certaine intensité. Il est donc indispensable que l'ouvrage ait une grande souplesse, pour suivre lentement, sans désorganisation sensible, les mouvements du sous-sol. Ainsi, cette règle absolue exclut, pour la constitution des barrages, l'emploi de matériaux en dur tels que maçonnerie de moellons ou massifs de béton.

La terre imperméable de marne et d'argile, bien débarrassée de toutes souches ou débris de végétaux, est l'élément essentiel de la retenue. Elle constitue l'écran qui s'oppose au passage des eaux. Pour cela, elle doit être montée sur un fond bien imperméable. Avant le montage de la digue. l'emplacement qui la recevra sera débarrassé de la couche de terre arable ; celle-ci sera soigneusement mise de côté pour recouvrir, par la suite, le parement extérieur de l'ouvrage.

Quand les digues (fig. I) ont I m. 5o de hauteur maximum, la terre imperméable, bien pilonnée ou corroyée, est le seul élément constitutif. Les talus doivent ne pas excéder une pente de 2 de hauteur pour 3 de largeur el être protégés dẹs effets de l'eau et des agents atmosphériques par du 
gazon soit semé soit mis en mottes. On peut utiliser les ray-grass et les diverses graminées des prairies à racines si possible traçantes et chevelues. En pratique, on sèmera des balayures de greniers à foin. Dans les parties les plus humides, les plus proches de l'eau et qui sont soumises au ressac, on plantera ou sèmera des carex et des roseaux et, dans l'eau, des plantes aquatiques telles que nénuphars myribphylles et potamots, qui serviront à protéger les. berges et la digue du batillage.

Signalons que le fond de l'étang doit toujours rester en terre naturelle pour donner une fertilité normale aux eaux de la retenue.

Pour les digues de plus grande importance, on peut utiliser d'autres matériaux sains, suivant les ressources de la région (terres légèrement

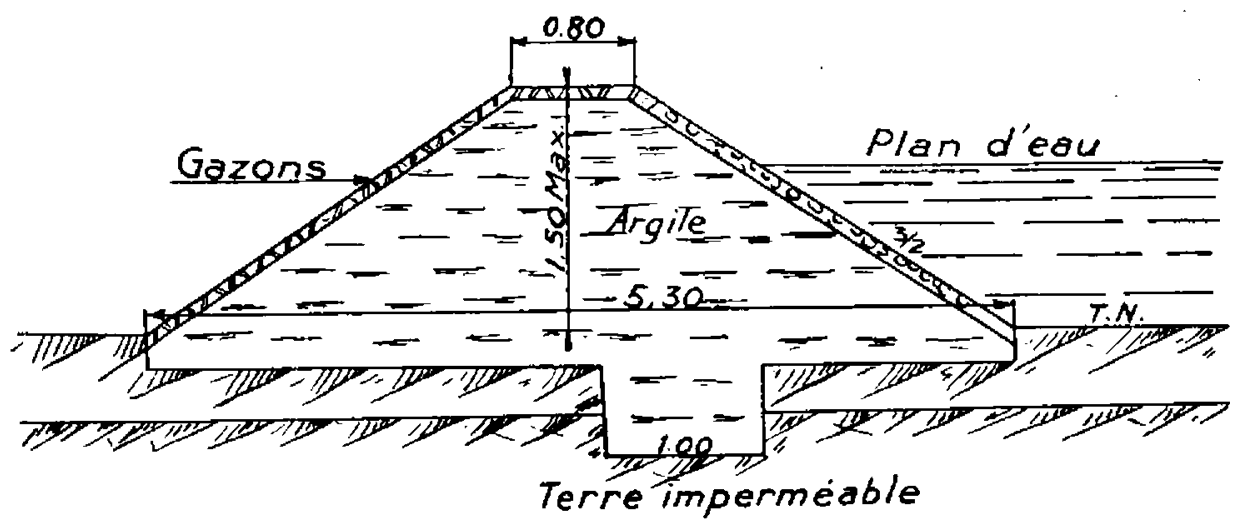

F10. 1

sableuses, graviers, cailloux), pour exécuter une partie des remblais ; mais il est obligatoire d'établir dans chaque cas un masque d'étanchéité en terre imperméable, sur toute la hauteur de l'ouvrage. La répartition des autres matériaux est fonction de leur nature et demande une étude très soignée par un spécialiste.

Il est indispensable d'apporter un soin particulier à la mise en place du remblai. Un remblai d'argile ne s'exécute bien que si le matériau employé garde un degré d'humidité constant et égal à celui qu'il avait avant son extraction. Pour cela, il faut employer les terres immédiatement après la fouille et, à l'occasion, les protéger du soleil, du gel ou de la pluie. Le pilonnage vu le corroyage par couches horizontales doit être poussé jusqu'à ce que la masse ait repris une densité égale ou presque à celle qu'elle avait en place. Le remblai ainsi exécuté doit être immédiatement protégé en surface par des gazons, comme il a été dit plus haut. Si la diguc est importante, le côté qui reçoit l'eau est recouvert par des cailloux et des moellons prosés à sec, les petỉs éléments contre l'argile et le gros en parement exté. rieur. 
Une digue, établie dans ces conditions, est longtemps stable, à condition d'être bien surveillée et entretenue.

Pour entretenir une digue de terre, il faut d'abord empècher le développement d'arbres, et même d'arbustes, dont les racines amorcent, avec le temps, des renards ou voies d'eau, dont l'importance croit rapidement après les premiers suintements. Ensuite, il est indispensable d'entretenir en bon état le chemin de dessus de digue, de façon que l'eau de pluie n'y séjourne jamais. La figure 2 montre les ravages qu'une ornière peut faire sur une digue de terre. L'eau stagnante se fraie un chemin dans l'argile et détache de la masse tout un bloc qui se désorganise, glisse et peut créer une rupture de digue. Quelques heures de travail tous les ans, pour reboucher,

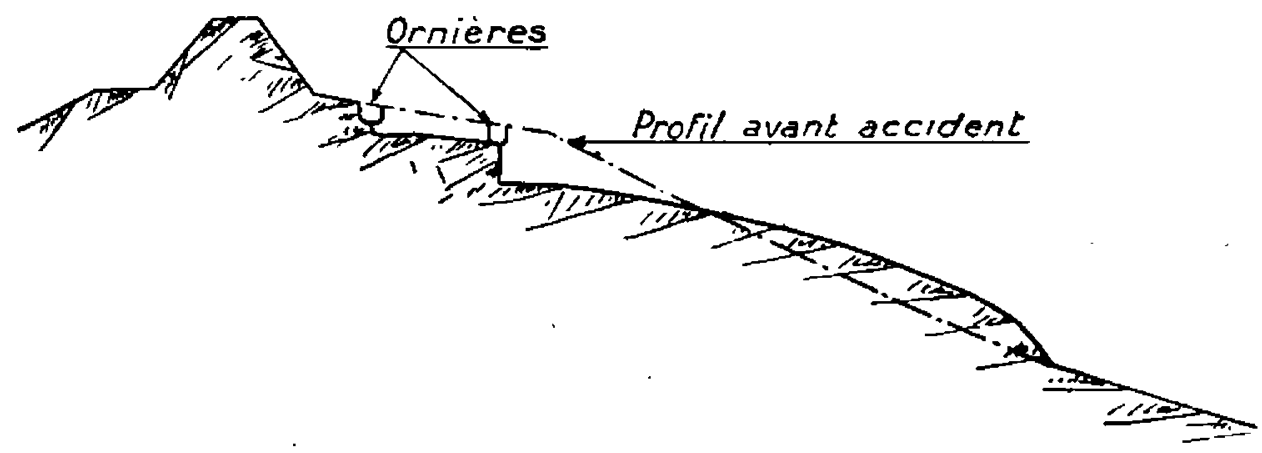

F10. 2

en terre saine, bien pilonnée, toutes les flaches, évitent les plus gros des accidents.

Le gazon doit être fauché ou brulé chaque année. Le pacage de bovins sur les talus n'est pas recommandable car les animaux font avec leurs pieds des trous qui nuisent au libre écoulement des eaux de ruissellement. ce qui crée des accidents de surface et nuit à la solidité de l'ensemble. Le pacage par les ovins est au contraire plutót favorable, car le piétinement des moutons produit un tassement du sol. On l'interrompra loutefois si les moutons en arrivent à dégrader les berges par arrachement des touffes d'herbe.

Par ailleurs, pour protéger les talus du batillage, il est utile, dans certains étangs soumis à des vents quelque peu importants, de faire flotter, à environ o $\mathrm{m}$. jo du bord, des baliveaux mis bout à bout. Ce dispositif qui casse les vagues constitue un excellent brise-lames.

Enfin, et surtout, il faut éviter de construire sur les digues des ouvrages en béton ou en matériaux durs dont la liaison avec l'argile est toujours mauvaise. Les murs de masque sont à bannir systématiquement. Il est aisé d'en faire la prewve et l'expérience confirme nos dires. 
On fait un beau mur qui recoupe au fond un terrain sain bien imper. méable. Il est très agréable à voir ; son courronnement est un chemin rêvé pour les tournées et pour les pêches. Il assure une étanchéité remarquable pendant quelques années. Puis, bientôt, on remarque des fissures et la terre ne colle plus à la paroi. Que s'est-il passé ? Tout simplement l'élément souple de la digue a suivi les mouvements plastiques du soussol, tandis que l'élément rigide est resté en suspens, retenu seulement en quelques points plus résistants. Et la catastrophe arrive bien vite : l'eau pénètre dans la digue, trouve un chemin de moindre résistance et c'est le classique renard. Surtout que l'on ne s'avise par de réparer en mettant du béton car, à moins de procéder par de conteuses injections, on ne réalise jamais un bon colmatage. On ne résoud pas le problème ; on le

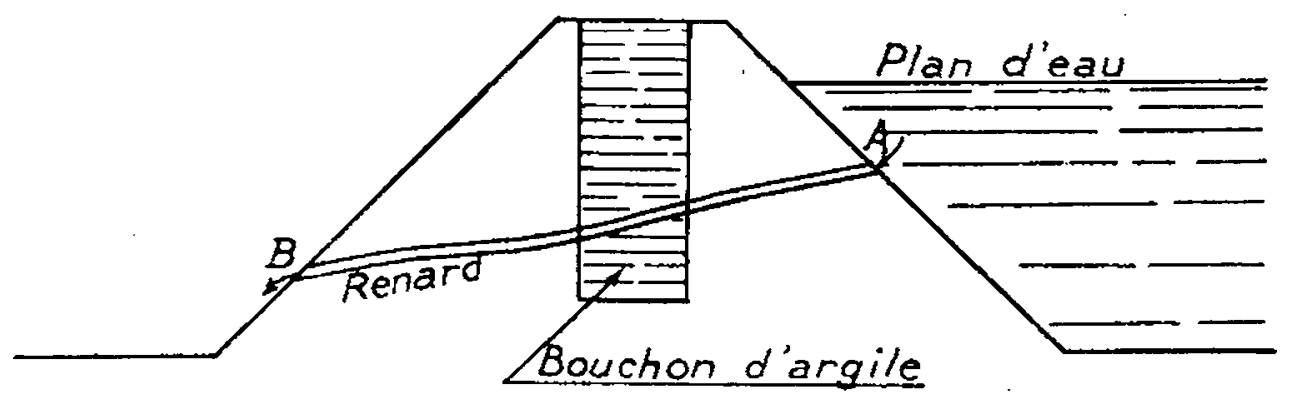

Fio. 3

reporte seulement de quelques années, car le même phénomène se reproduira 2 ou 3 ans plus tard.

Il n'existe qu'un moyen simple pour combattre les voies d'eau : c'est d'utiliser le seul matériau qui a une bonne liaison avec celui qui est en place et qui suivra toutes les déformations. Il faut construire ou rétablir un écran soigné en terre imperméable. Mais attention de ne pas ouvrir la digue (à l'occasion d'un assec) sur toute la longueur AB (fig. 3). Aussi soigné que soit le remblai, il se créerait aux liaisons entre la terre en place et la terre rapportée des points de moindre résistance qui constitueraient pour l'eau un passage possible. Il faut au dontraire faire une tranchée de direction, sensiblement perpendiculaire au sens de la voie d eau, c'est-à-dire parallèle à l'axe de la digue. Ensuite, on réparera lés sumlements, on les recoupera très largement dans la bcrne terre en s'étendant en longueur et en profondeur et on rebouchera la Iranchée bien soigneusement. On aura ainsi créé un obstacle efficace au jiassage de l'eau ciui ne s infiltrera plus si on a fait convenablement le travail.

Il y a malheureusement des ouvrages en matériaux durs dont l'installation ne peut être évitée : ce sont les déversoirs de crues et les dispositifs 
de vidange. Il faut rechercher les moyens de les placer en réduisant au maximum les ennuis qui peuvent en résulter.

Les déversoirs de crues ne sont pas très gènants. Ils se placent aux extrémités des digues, à un endroit où elles sont basses ef ne supportent pas de grosses pressions. Parfois on peut - et il ne faut pas manquer de le faire - les placer avec un léger aménagement des abords, en dehors de la digue. Le sol à l'amont et à l'aval du déversoir devra être protégé des entraînements dus aux courants d'eau, par des revêtements constitués de dalles en béton de I mètre carré de surface maximum, mises côte à côte, ou par de gros moellons posés à sec. Tous les ans, il sera nécessaire

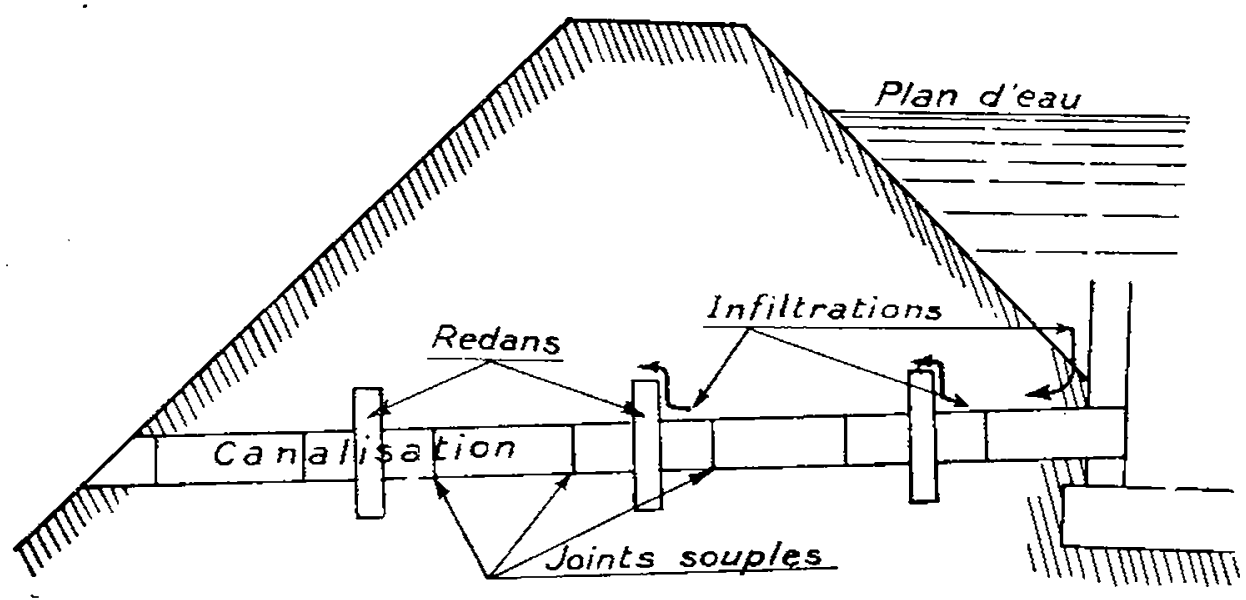

FIG. 4

de vérifier les terrains à la liaison avec le déversoir el de faire disparaitre tout décollement et toutes fissures.

Afin d'éviter qu'aù moment d'une crue les roseaux faucardés poussés par le vent ne viennent s'accumuler contre la grille du déversoir ct la bouchent, on prendra la simple et peu coûteuse précaution de les arrêter par des piquets plantés par o $\mathrm{m}$. 30 à $\circ \mathrm{m}$. 50 de fond, distant entre eux de o m. 20 et disposés en arc de cercle à quelques mètres du déversoir.

Les ouvrages de restitution ou de vidange sont plus délicats à établir. On ne peut se dispenser d'installer sous la digue, au point le plus bas, c'est-à-dire là où sont les plus fortes pressions, un exutoire pour l'eau. Le choix de la conduite importe peu ; cc qui est intéressant, c'est qu'elle soit constitué d'éléments de faible lonqueur liés entre eux par des joints étanches et souples (en goudron ou en caoutchouc par exemple), pour que la canalisation épouse les variations dues aux mouvements du terrain, au poids des terres et aux surcharges. De plus, pour gêner le passage de !'eau à la liaison entre les terres de remblai et la conduite, il est utile de 


\section{Coupe AB}

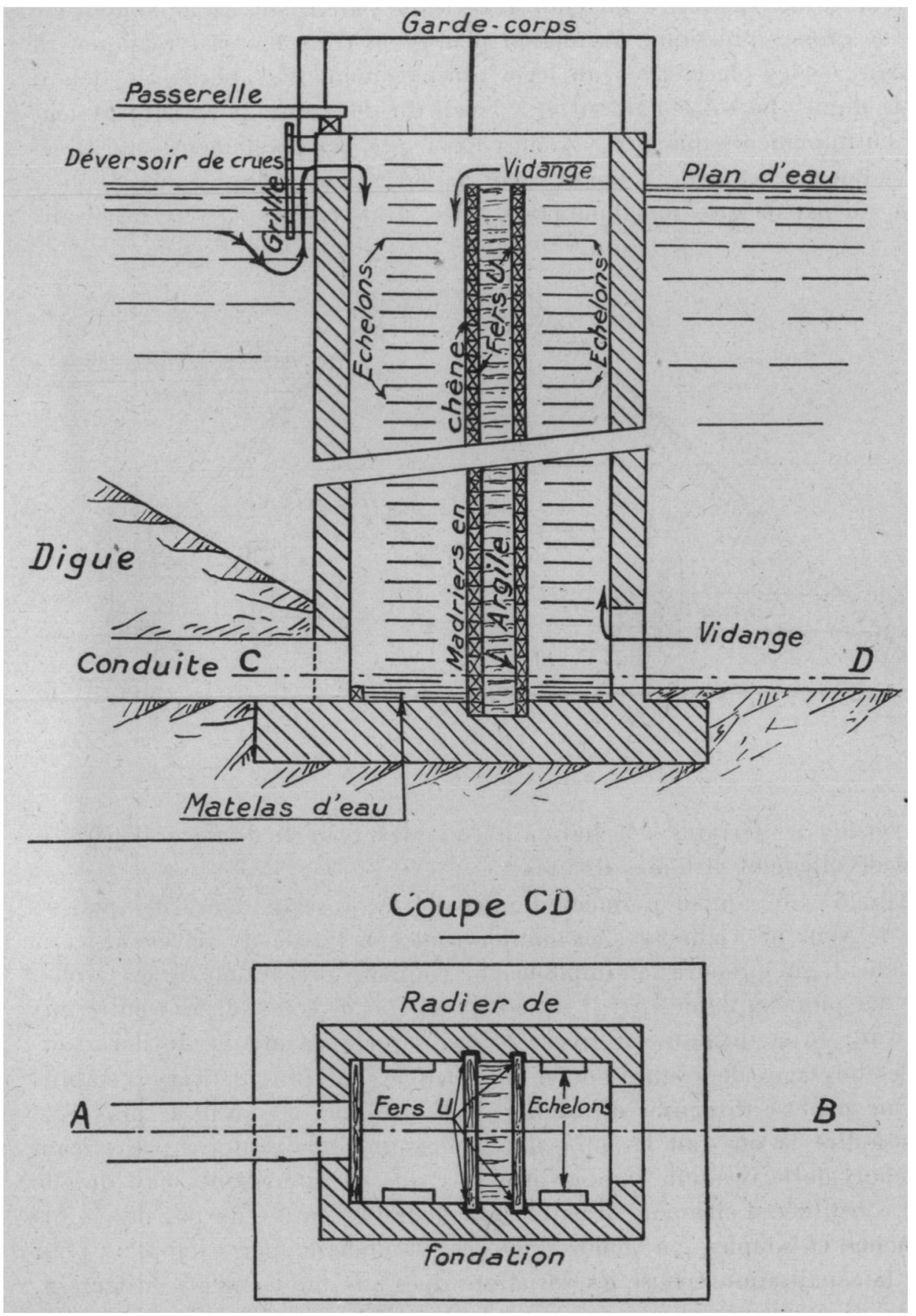


solidariser à celle-ci, de place en place, des redans dont le but est de diminuer, avec la longueur accrue du cheminement, la pression de l'eau et partant de restreindre les risques de fuites (fig. 4).

Un problème important esł celui de l'installation du dispositif de prise d'eau dans le réservoir, à l'origine de la canalisation. De nombreux systèmes sont en service, plus ou moins satisfaisants pour la pisciculture. A notre avis, le meilleur dispositif est le suivant :

A l'extrémité amont de la canalisation de vidange, juste au pied de la digue, s'installe (fig. 5) une tour parallèlépipédique en béton ou en maçonnerie de moellons qui repose sur un radier en béton suffisamment large pour soutenir l'ensemble, sans faire travailler, sous l'action des charges et surcharges, à plus de o kg. 500 au centimètre carré, l'argile de sous-sol. La canalisation de vidange débouche dans cette tour, à la hauteur du fond de l'étang.

Un batardeau en madriers partage la tour en deux chambres communiquant librement, l'une avec l'étang, l'autre avec la conduite de vidange. Quatre fers en $\mathrm{U}$ sont scellés dans les maçonneries qui reçoivent deux rangées de madriers en chêne placés l'un sur l'autre, distantes de o $\mathrm{m}$. 3o.

Dans cet intervalle, on tasse de l'argile; de sarte que l'on obtient un voile parfaitement étanche que l'on monte jusqu'à la hauteur de la retenue. Une étanchéité parfaite est obtenue en jetant, au fur et à mesure du remplissage, de la poussière de charbon de bois (fraisil) dans l'eau, juste devant les madriers. Les filets d'eau entraînent ce poussier qui colmatera les points de contact des madriers entre eux et des madriers avec les fers en U. On aura auparavant bourré de mousse les joints entre les madriers et les fers en $U$.

Pour la vidange qui s'opère par déversemẹt par-dessus ce voile, il suffit d'enlever successivement chacun des lits de madriers. De plus, sur les parois verticales de la tour, côté canalisation, on aménage à la cote maximum de l'étang un déversoir de crues protégé $\mathbf{a}$ o $\mathrm{m}$. 3o de la paroi par une grille de $\mathrm{I}$ mètre de haut plongeant de o $\mathrm{m}$. 5o dans l'eau dont le but est de retenir dans l'étang feuilles mortes, herbes faucardées ou corps flottants. C'est ainsi que les bancs d'herbes faucardées peuvent venir se coller contre la grille, sans s'opposer au passage de l'eau qui se fait par-dessous.

Un madrier mobile obstruera la partie inférieure de la conduite d'évacuation et retiendra ro à 20 centimètres d'eau au fond de la tour qui feront matelas protégeant le béton de l'érosion que pourrait provoquer la chute d'eau des déversoirs supérieurs. Enfin, au sommet de la tour, repose l'une des extrémités d'une passerelle de manœuvre qui permet l'accès depuis la digue. Un garde-corps facilite l'utilisation des échelons scelles dans les murs verticaux indispensables pour la mise en place et la dépose ues madriers, 
Il est fort difficile de donner un prix de revient de cet ouvrage car les prix de construction en étang sont fonction de nombreux facteurs particuliers inestimables a priori : ressources du pays en matériaux, en ouvriers, éloigrnement de la route, facilités d'accès. Mais il n'est pas plus onéreux que la plupart des dispositifs habituellement adoptés avec bondes de fond, crics de mancuvres et murs de protection en retour.

Au point de vue piscicole, il présente par ailleurs les gros avantages suivants :

- Fermeture étanche de l'eau ;

- Réglage facile du niveau de l'étang ;

- Vidange facile de l'étang par tranches successives évitant tous les inconvénients de la vidange par le fond;

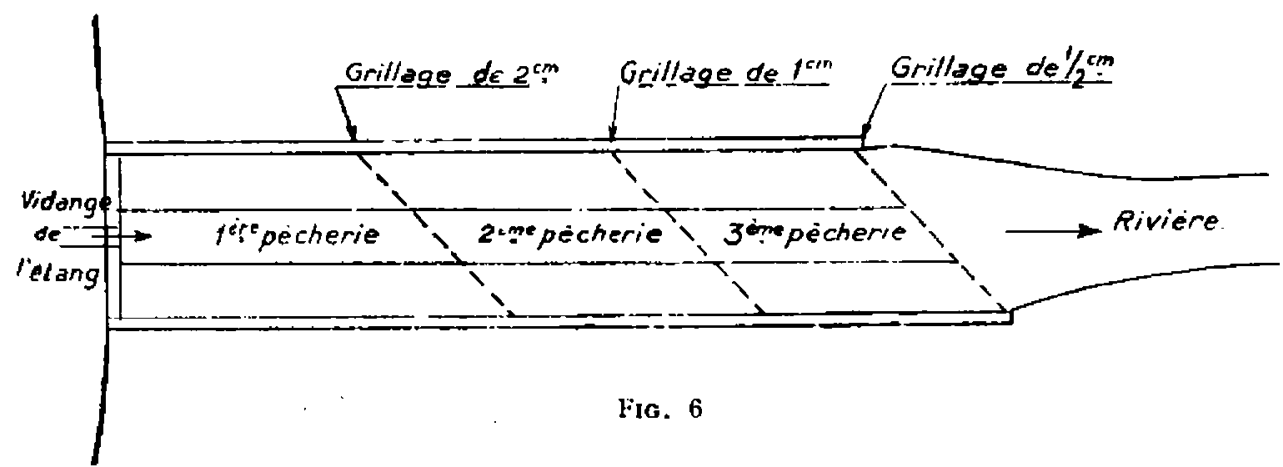

- Evacuation, pendant la saison de croissance du poisson, de l'eau du fond de l'étang, conservant ainsi les eaux de surface chaudes et riches en plancton ;

- Economie d'achat d'une vanne métallique de fond et économie de construction d'un déversoir de côté.

Il y aura intérêt le plus souvent à creuser dans le fond de l'étang une poêle à bords droits de 20 à 30 centimètres de profondeur dont le fond sera au niveau ou un peu au-dessus de la conduite de vidange et où le poisson sera rassemblé lors de la pêche pour être éclusé par coups d'eau successifs dans la pêcherie aval.

Des tours de prise d'eau de cette nature ont été étudiées et construites aux étangs du Der. Elles donnent à l'usage entière satisfaction.

Pour terminer cet examen des ouvrages des étangs, il faut dire un mot des conditions d'une bonne installation de la pècherie. Sauf impossibilité absolue, il est indiqué de l'installer à l'aval de la digue. Ainsi, les manœuvres peuvent s'effectuer dans une eau relativement propre et les poissons pêchés ne sont pas inutilement fatigués par la vase remuée par les pècheurs. De plus, la situation aval rapproche les pêcheurs de la route utilisée pour l'enlèvement des produits et évite au personnel les remontées pénibles de la digue. 
Nous proposons le modèle suivant :

Dès la sortie de la canalisation de vidange, que l'on pourra obstruer après le passage des poissons par un grillage, on établit un bassin bétonné qui devra supporter une nappe d'eau de o $\mathrm{m}$. 6o environ et dont les dimensions variables avec la capacité des étangs seront de 2 à 4 mètres pour la largeur et de 8 à r 2 mètres pour la longueur.

Ce bassin, dans lequel on pêchera à l'épuisette, devra être divisé en trois parties (voir fig. 6) dans le sens de la longueur, par des grilles de mailles différentes : 2 centimètres pour la première ; I centimètre pour la seconde ot $\mathrm{r} / \mathrm{a}$ centimètre pour la dernière, placées de telle sarte que pour une même hauteur d'eau la section d'écoulement à travers les grilles

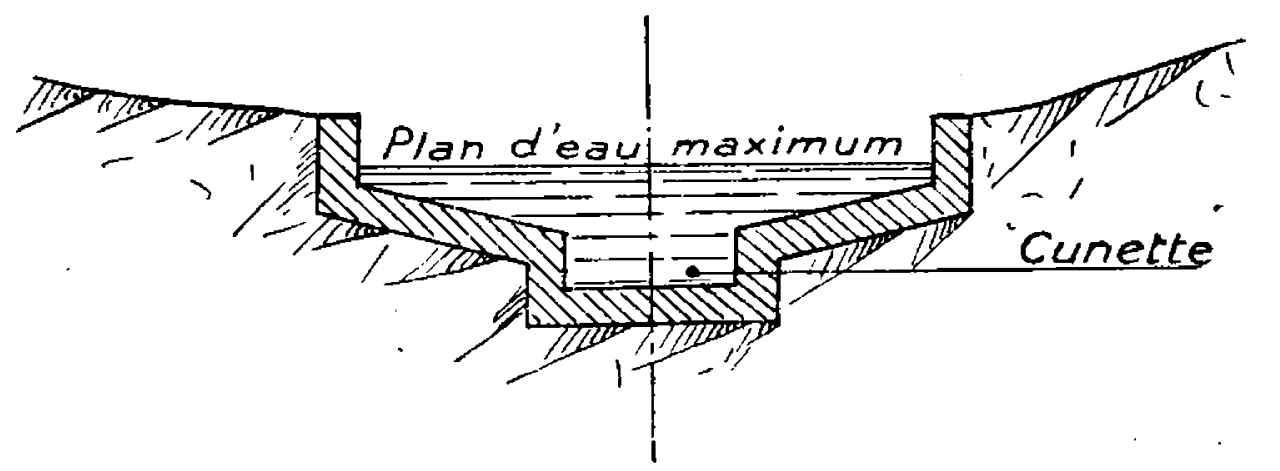

Fig. 7

soit une demi-fois plus grande que la section transversale normale de la rivière d'aval. (ll faut que la section calculée soit plus grande que la section d'ecoulement, car à l'usage les grilles s'obstruent partiellement.)

Ce dispositif a l'avantage de favoriser un tri automatique par grosseur, ce qui facilite notablement les opérations du triage. Si l'ouvrage doit servir pour l'extraction des produits d'un étang important (5o hectares), on aura intérêt à utiliser à chaque séparation du bassin un jeu de deux grilles mobiles de méme calibre. L'un des jeux étant en place et partiellement bouché, on placera le second jeu immédiatement à côté du premier que l'on pourra retirer et nettoyer. Dans les établissements où l'on néglige la récupération des alevins, on pourra se contenter de diviser la pêcherie en deux parlies en n'utilisant que les grilles de $\dot{2}$ centimètres et I centimètre.

A l'aval du bassin, on pourra installer dans la rivière un batardeau mobile qui règlera la hauteur de l'eau dans la pêcherie. Le fond de cette pêcherie sera établi suivant le profil de la fig. 7 qui comporte une cunette centrale. Celle-ci permet une concentration plus grande de poissons sous une faible hauteur d'eau, ce qui est particulièrement utile en fin de pêche. 
Je ne saurais mieux conclure cet article qu'en insistant une nouvelle fois auprès des propriétaires et des exploitants pour leur rappeler les principes suivants que noug estimons essentiels :

- Banissez de vos digues en terre tout matériau dur ;

- Réparez immédiatement le moindre affaissement, la muindre fissure ;

- Dégagez vos digues de toute végétation importante et préservez-vous soigneusement du batillage ;

- Défiez-vous des aménagements à manœuvres compliquées ct qui n'ont pas fait leurs preuves ;

- Soyez vigilants, examinez souvent vos ouvrages, entretenez-les, préservez votre fonds, c'est le seul moven de le faire normalement produire. 